\title{
Sociodemographic and geographical inequalities in under- and overnutrition among children and mothers in Bangladesh: a spatial modelling approach to a nationally representative survey
}

\author{
Mohammad Nahid Mia ${ }^{1, *}$, M Shafiqur Rahman ${ }^{2}$ and Paritosh K Roy ${ }^{2}$ \\ ${ }^{1}$ Health Systems and Population Studies Division, International Centre for Diarrhoeal Disease Research, Bangladesh \\ (icddr,b), 68 Shaheed Tajuddin Ahmed Sarani, Mohakhali, Dhaka 1212, Bangladesh: ${ }^{2}$ Institute of Statistical \\ Research and Training, University of Dhaka, Dhaka, Bangladesh
}

Submitted 25 July 2017: Final revision received 4 March 2018: Accepted 21 March 2018; First published online 2 May 2018

\begin{abstract}
Objective: To investigate the sociodemographic and geographical variation in under- and overnutrition prevalence among children and mothers.

Design: Data from the 2014 Bangladesh Demographic and Health Survey were analysed. Stunting and wasting for children and BMI $<18.5 \mathrm{~kg} / \mathrm{m}^{2}$ for mothers were considered as undernutrition; overweight was considered as overnutrition for both children and mothers. We estimated the prevalence and performed simple logistic regression analyses to assess the associations between outcome variables and predictors. Bayesian spatial models were applied to estimate region-level prevalence to identify the regions (districts) prone to under- and overnutrition.

Settings/Subjects: Children aged $<5$ years and their mothers aged $15-49$ years in Bangladesh.

Results: A significant difference $(P<0 \cdot 001)$ was observed in both under- and overnutrition prevalence between poor and rich. A notable regional variation was also observed in under- and overnutrition prevalence. Stunting prevalence ranged from $20.3 \%$ in Jessore to $56.2 \%$ in Sunamgonj, wasting from $10.6 \%$ in Dhaka to $19.2 \%$ in Bhola, and overweight from $0.8 \%$ in Shariatpur to $2.6 \%$ in Dhaka. Of the sixty-four districts, twelve had prevalence of stunting and thirty-two districts had prevalence of wasting higher than the WHO critical threshold levels. Similarly, fifty-three districts had prevalence of maternal underweight higher than the national level. In contrast, the prevalence of overweight was comparatively high in the industrially equipped metropolitan districts.

Conclusions: Observed sociodemographic and geographical inequalities imply slow progress in the overall improvement of both under- and overnutrition. Therefore, effective intervention programmes and policies need to be designed urgently targeting the grass-roots level of such regions.
\end{abstract}

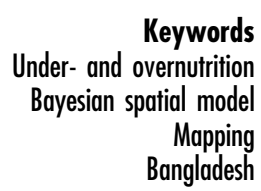

Undernutrition is a serious public health problem with important consequences for survival, cognitive development and optimum physical growth ${ }^{(1-3)}$. It is also considered one of the causes of non-communicable diseases, morbidity, and decreased economic productivity and earning potential ${ }^{(1,4,5)}$. These problems tend to be severe among children younger than 5 years and women of reproductive age, particularly in developing countries struggling to emerge from the scourge of extreme poverty $^{(6)}$. Undernutrition causes $3 \cdot 1$ million child deaths annually, accounting for $45 \%$ of total child deaths in $2011^{(1,7)}$. Although the global prevalence of undernutrition is continuing to reduce in both children and women, most progress has been made in South-East Asia including
Bangladesh, in comparison with slow progress in sub-Saharan Africa $^{(8-10)}$. Similarly, overnutrition (overweight or obesity) is also a public health concern because overweight children are at greater risk of developing many health problems, like overweight adults, such as heart disease, respiratory illness and type 2 diabetes ${ }^{(11)}$. Moreover, overnutrition has a negative effect on children's social and emotional well-being and self-esteem, resulting in impaired social development.

Bangladesh has been commended worldwide for its recent gains in health and nutrition. The prevalence of childhood stunting (low height-for-age) and underweight (low weight-for-age) has decreased from 63 and $62 \%$ in 1990 to 42 and $32 \%$ in 2013, respectively ${ }^{(12)}$. 
The prevalence appears to be lower than in Pakistan (which had stunting and underweight prevalence of 45 and $32.6 \%$, respectively, in 2012), but still higher than in India (39 and 29\%, respectively, in 2014) ${ }^{(12)}$. Despite more than a decade of intervention and great socio-economic development, however, Bangladesh still has a large number of children with stunting ( 6 million) and wasting $(2.4 \text { million })^{(13)}$. In the review of the Millennium Development Goals, the country was criticized for not focusing on nutrition issues appropriately and it failed to achieve the Millennium Development Goal 1 target of child underweight prevalence of $31 \%$ by $2015^{(14,15)}$. Similarly, the prevalence of women with underweight (BMI $<18.5 \mathrm{~kg} / \mathrm{m}^{2}$ ) has decreased from $53 \%$ in 1996 to $19 \%$ in 2014. However, the rate is not lower than those of neighbouring countries, such as India, Nepal and Pakistan $^{(12)}$. In developing countries like Bangladesh, overweight or obesity is also a growing concern. In Bangladesh, the proportion of women with overweight $\left(\geq 25.0 \mathrm{~kg} / \mathrm{m}^{2}\right)$ has increased from $3 \%$ in 1996 to $24 \%$ in $2014^{(14)}$. The rate of increase is faster than in India $(10 \cdot 6 \%$ in 1999 to $20.2 \%$ in 2016) and Nepal (9.0\% in 2006 to $22 \cdot 0 \%$ in 2016) ${ }^{(16-19)}$. The most recent public health concern is childhood overweight or obesity because the prevalence has increased at an alarming rate, particularly in urban areas ${ }^{(20)}$. Thus, identifying the reasons behind the slow progress of undernutrition and the drastic increase of overnutrition prevalence has become a challenge for public health researchers. Studies conducted in the last decade have identified maternal education, socio-economic status, short birth spacing, food insecurity and low birth weight as potential factors associated with undernutrition $^{(21-25)}$.

Over the last decade, Bangladesh has demonstrated significant improvement on each of these fronts; nevertheless, progress might not be the same for all regions of the country ${ }^{(26)}$. For instance, urban people are in better condition concerning many sociodemographic aspects compared with their rural counterparts, where about $75 \%$ of the population resides ${ }^{(27)}$. Therefore, it is essential to scrutinize the regional variation and identify the regions (districts) with high prevalence where imperative actions are required for quick improvement. Similarly, studies have indicated that the most common factors associated with overnutrition (overweight or obesity) are the imbalance between food intake and energy expenditure and low physical activity ${ }^{(28,29)}$. However, food habits and types of physical activity vary across the regions of the country, particularly between urban and rural areas. Therefore, it is also necessary to identify the regions with high prevalence of overweight or obesity for designing interventions to prevent overweight or obesity.

Bangladesh is the seventh most populous country in the world with a population of 164 million and is projected to be the eighth most populous (202 million) by $2050^{(30)}$. Administratively, the country is divided into division ( $n 8)$, district ( $n$ 64), upazila ( $n$ 489)/thana ( $n$ 631) and union ( $n$ 4553) ${ }^{(27)}$. Notwithstanding the recent robust annual growth in gross domestic product, averaging $6 \%$ per annum between 2011 and $2013^{(31)}$, the country remains one of the poorest in the world with $32 \%$ (rural $52 \%$, urban $22 \%$ ) of the population living below the poverty line of \$US 1.25 per day ${ }^{(27)}$. The country has characteristics similar to a typical developing nation with high unemployment rate (29\%) and highly skewed income inequality ${ }^{(27,32)}$. Twenty per cent of the richest among the population owns about $42 \%$ of the wealth, while the poorest $20 \%$ of the population owns $9 \%$ of the wealth ${ }^{(33)}$. Besides, the distribution of rich and poor is not the same for all regions of the country as a majority of rich people live in urban areas in comparison with their rural counterparts ${ }^{(33)}$.

Bangladesh is unique among low- and middle-income countries in having valid and nationally representative household survey-based statistical evidence for only national and divisional levels; there are very limited statistics or data available for sub-division or district level. Additionally, research conducted to assess the regional inequalities in health and nutrition has been very limited. The issue of geographical differences in undernutrition as well as overnutrition has emerged as a matter of concern considering growing evidence of the strong persistence of existing inequalities ${ }^{(8,34)}$. However, national analyses, although useful, have little programmatic value for advocacy because they fail to highlight the extent of geographical inequalities that is essential for designing appropriate interventions and policies on nutrition and health. Thus, the focus of the present study was to investigate the nutritional status of women and children at the district level to identify the vulnerable districts and to understand the full extent of geographical and social inequalities in relation to sociodemographic variables. We also analysed data about maternal demographic factors to understand the social determinants of both under- and overnutrition. The findings from the study will help policy makers design appropriate policies and programmes to reduce the burden of under- and overnutrition and to achieve Sustainable Development Goal 2.2.

\section{Materials and methods}

\section{Study design and participants}

The data for the present study were extracted from the 2014 Bangladesh Demographic and Health Survey (BDHS) conducted between July and November 2014. The BDHS is based on a two-stage stratified cluster sampling scheme to provide a representative sample of Bangladesh as a whole. In the first stage, 600 enumeration areas (EA), the primary sampling units or clusters, were selected from the list of EA provided by the Bangladesh Bureau of Statistics with probability proportional to EA size, which ensured 207 EA in urban areas and 393 EA in rural areas. 
In the second stage of sampling, a systematic sample of approximately thirty households was selected per cluster or primary sampling unit. Details of the sampling design are available in the main BDHS report ${ }^{(14)}$. A total of 18245 ever-married women of childbearing age were selected for the survey and finally 17863 were interviewed. Among them, 10930 did not have any children younger than 5 years and hence were excluded from the analysis. We also excluded 320 women who had given birth in the two months preceding the survey. A total of 6933 children aged 0-59 months and their mothers aged 15-49 years were included in the analysis.

\section{Definition of variables}

\section{Dependent variables}

Stunting (low height-for-age), wasting (low weightfor-height) and overweight (high weight-for-height) were considered the outcome variables for children. The first two were used to define undernutrition; the last one to define overnutrition. Underweight (low weight-for-age) is also often considered an undernutrition indicator among children; however, weight-for-age is a composite index of height-for-age and weight-for-height and thus is well captured by stunting and wasting. Therefore we did not use underweight for children. $Z$-scores for each of the anthropometric indices were calculated using the median of the reference population in the 2006 WHO child growth standards $^{(35)}$. Children with $Z$-score for height-for-age below -2 were classified as stunted. Similarly, children with $Z$-score for weight-for-height below -2 or above +2 were considered wasted or overweight, respectively. For women, the outcome variables were underweight and overweight. Women with $\mathrm{BMI}<18.5 \mathrm{~kg} / \mathrm{m}^{2}$ and $\mathrm{BMI} \geq 25.0 \mathrm{~kg} / \mathrm{m}^{2}$ were considered underweight and overweight, respectively ${ }^{(36)}$.

\section{Independent variables}

The socio-economic and demographic information of the children and women were considered as independent variables. These included age, sex, residence (rural, urban), mother's education (years of schooling: none, 1-5 years, 6-9 years, $\geq 10$ years), mother's BMI (underweight if $\mathrm{BMI}<18.5 \mathrm{~kg} / \mathrm{m}^{2}$ ), membership of any nongovernmental organization (yes, no) and wealth index (poor, poorest, middle, rich and richest). The wealth index was calculated, as described in the BDHS 2014 report $^{(14)}$, using principal component analysis of assets owned by households ${ }^{(37,38)}$. The score was then divided into five equal quintiles, with the first representing the poorest $20 \%$ and the fifth representing the richest $20 \%{ }^{(39,40)}$. More details are available elsewhere ${ }^{(14)}$.

\section{Statistical analyses}

As a bivariate analysis, the prevalence of under- and overnutrition in both children and women (assessed by the four indicators mentioned above), with 95\% CI, was estimated according to each of the individual-level predictors. A simple logistic regression model was performed to assess the association of under- and overnutrition with each of the background characteristics, separately; only the $P$ value from the likelihood ratio test of the model is reported to determine the significance of the association. All these analyses were performed controlling for the effect of the complex survey design using Stata's 'SVY' command. Further, three different multivariable logit models for each nutrition indicator with all predictors (background factors) found to be significant in the simple logistic regression analyses were fitted, to assess the net association of each predictor with the outcome controlling for the effects of other predictors and to estimate the district-level prevalence of underand overnutrition. Model 1 is a standard logit model with individual-level predictors. Model 2 is a multilevel logit model with individual-level predictors in the first level and district-level independently and identically distributed random effects in the second level. Model 3 is also a multilevel logit model with individual-level predictors in the first level, district-level both spatial (structured) and random effects on the second level. The reason for introducing a random effect in Model 2 was to capture the unobserved heterogeneity between the districts. The neighbouring structure at the district-level Bangladesh lattice motivated us to extend Model 2 to Model 3 by adding another random effect to capture the unobserved variations due to the district-level spatial structure. To introduce the spatial effects in Model 3, we used the intrinsically conditional autoregressive modelling approach $^{(41)}$ with hierarchical specification at the second stage $^{(42)}$. We estimated the models' parameters within the Bayesian framework with non-informative prior using the integrated nested Laplace approximation approach for summarizing the posterior distributions. Finally, the fitted model was used to predict the posterior probability of under- and overnutrition for an individual in a district. The district-level prevalence of under- and overnutrition was then estimated as the weighted average of the individual-level predicted probabilities, weighted by the individual sampling weights calculated based on the BDHS 2014 survey design, with $95 \% \mathrm{CI}^{(14)}$. The statistical software package Stata for Windows version 14.2 and the statistical software $\mathrm{R}$ version 4.3 .1 were used for all analyses.

\section{Results}

\section{Nutritional status of the children}

Table 1 shows the prevalence of child under- and overnutrition with $95 \%$ CI. Overall, the prevalence of stunting, wasting and overweight among the children aged $<5$ years was $36 \cdot 2,14 \cdot 3$ and $1.3 \%$, respectively. Children 
Table 1 Prevalence (\%) of undernutrition (stunting and wasting) and overnutrition (overweight) by sociodemographic variables among children aged 0-59 months ( $n$ 6933), Bangladesh, 2014

\begin{tabular}{|c|c|c|c|c|c|c|c|}
\hline \multirow[b]{2}{*}{ Variable } & \multirow[b]{2}{*}{$n$} & \multicolumn{2}{|c|}{ Stunting* } & \multicolumn{2}{|c|}{ Wasting† } & \multicolumn{2}{|c|}{ Overweightł } \\
\hline & & $\%$ & $95 \% \mathrm{Cl}$ & $\%$ & $95 \% \mathrm{Cl}$ & $\%$ & $95 \% \mathrm{Cl}$ \\
\hline \multicolumn{8}{|l|}{ Place of residence } \\
\hline Urban & 2181 & $30 \cdot 7$ & $27 \cdot 4,34 \cdot 2$ & $12 \cdot 2$ & $10 \cdot 5,14 \cdot 1$ & $2 \cdot 1$ & $0.9,1.8$ \\
\hline Rural & 4752 & 38.0 & $36 \cdot 0,40 \cdot 1$ & $15 \cdot 1$ & $13 \cdot 7,16 \cdot 5$ & $1 \cdot 3$ & $1.5,3.0$ \\
\hline$P$ value & & \multicolumn{2}{|c|}{$<0.001$} & \multicolumn{2}{|c|}{$<0.001$} & \multicolumn{2}{|c|}{0.021} \\
\hline \multicolumn{8}{|l|}{ Child's age (months) } \\
\hline $0-5$ & 554 & $14 \cdot 1$ & $10 \cdot 0,19 \cdot 8$ & $20 \cdot 1$ & $15 \cdot 3,25 \cdot 8$ & $1 \cdot 2$ & $0.5,3.0$ \\
\hline $6-11$ & 782 & $19 \cdot 6$ & $16 \cdot 4,23 \cdot 1$ & $18 \cdot 0$ & $14 \cdot 5,22 \cdot 0$ & 1.7 & $1.0,3.0$ \\
\hline $12-17$ & 765 & 31.0 & $26 \cdot 3,36 \cdot 1$ & $17 \cdot 4$ & $14 \cdot 0,21 \cdot 5$ & $2 \cdot 1$ & $1 \cdot 2,3 \cdot 8$ \\
\hline $18-23$ & 685 & $46 \cdot 6$ & $40 \cdot 5,52 \cdot 8$ & $12 \cdot 4$ & $9 \cdot 8,15 \cdot 7$ & $2 \cdot 2$ & $1 \cdot 2,3 \cdot 8$ \\
\hline$\geq 24$ & 4147 & 41.6 & $39 \cdot 2,44.0$ & $12 \cdot 6$ & $11.4,13.9$ & $1 \cdot 3$ & $0.8,1.8$ \\
\hline $\bar{P}$ value & & \multicolumn{2}{|c|}{$<0.001$} & \multicolumn{2}{|c|}{$<0.001$} & \multicolumn{2}{|c|}{0.147} \\
\hline \multicolumn{8}{|l|}{ Child's sex } \\
\hline Male & 3552 & $36 \cdot 9$ & $34.8,38.9$ & $15 \cdot 1$ & $13 \cdot 2,17 \cdot 1$ & 1.5 & $1 \cdot 1,2 \cdot 1$ \\
\hline Female & 3381 & $35 \cdot 4$ & $33.0,37.9$ & $13 \cdot 5$ & $12 \cdot 2,15 \cdot 1$ & 1.5 & $1 \cdot 1,2 \cdot 0$ \\
\hline$P$ value & & \multicolumn{2}{|c|}{0.067} & \multicolumn{2}{|c|}{0.053} & \multicolumn{2}{|c|}{0.813} \\
\hline \multicolumn{8}{|c|}{ Mother's age at first birth (years) } \\
\hline$\leq 19$ & 5064 & $38 \cdot 7$ & $36 \cdot 6,40 \cdot 7$ & 14.6 & $13 \cdot 2,16 \cdot 1$ & $1 \cdot 3$ & $1.0,1.8$ \\
\hline $20-29$ & 1806 & 29.5 & $26 \cdot 7,32 \cdot 4$ & 13.6 & $11.7,15.8$ & $2 \cdot 0$ & $1.4,2.9$ \\
\hline$\geq 30$ & 63 & $25 \cdot 4$ & $13 \cdot 8,42 \cdot 0$ & 11.7 & $3 \cdot 7,31 \cdot 4$ & 0.7 & $0.2,4.9$ \\
\hline $\bar{P}$ value & & \multicolumn{2}{|c|}{$<0.001$} & \multicolumn{2}{|c|}{0.025} & \multicolumn{2}{|c|}{0.042} \\
\hline \multicolumn{8}{|l|}{ Mothers' height $(\mathrm{cm})$} \\
\hline$\leq 150$ & 3410 & 45.9 & $43.5,48.4$ & $15 \cdot 1$ & $13 \cdot 4,17 \cdot 0$ & $1 \cdot 3$ & $0.9,1.9$ \\
\hline$>150$ & 3523 & $26 \cdot 4$ & $24.4,28.5$ & 13.6 & $12 \cdot 3,15 \cdot 0$ & 1.7 & $1 \cdot 2,2 \cdot 3$ \\
\hline$P$ value & & \multicolumn{2}{|c|}{$<0.001$} & & & & \\
\hline First birth child & & & & & & & \\
\hline Yes & 2685 & 31.6 & $29 \cdot 3,34 \cdot 1$ & $14 \cdot 1$ & $12 \cdot 3,16 \cdot 0$ & 1.7 & $1 \cdot 2,2 \cdot 4$ \\
\hline No & 4248 & 39.0 & $36 \cdot 9,41 \cdot 2$ & 14.5 & $13 \cdot 2,15 \cdot 9$ & 1.4 & $1.0,1.9$ \\
\hline$P$ value & & & & & & & \\
\hline Mother's education & & & & & & & \\
\hline Illiterate & 1071 & $47 \cdot 3$ & $42 \cdot 3,52 \cdot 3$ & 14.7 & $12 \cdot 3,17 \cdot 5$ & 0.7 & $0.3,1.5$ \\
\hline $1-5$ years of schooling & 1924 & $43 \cdot 8$ & $40 \cdot 9,46 \cdot 8$ & $15 \cdot 3$ & $13 \cdot 2,17 \cdot 6$ & $1 \cdot 1$ & $0.7,1 \cdot 8$ \\
\hline $6-9$ years of schooling & 3206 & $31 \cdot 0$ & $29 \cdot 0,33 \cdot 1$ & 13.9 & $12 \cdot 5,15 \cdot 5$ & 1.6 & $1 \cdot 2,2 \cdot 3$ \\
\hline$\geq 10$ years of schooling & 732 & $19 \cdot 7$ & $16 \cdot 4,23.5$ & $12 \cdot 8$ & $10 \cdot 3,15 \cdot 8$ & $3 \cdot 6$ & $2 \cdot 2,5 \cdot 7$ \\
\hline$P$ value & & & & & & & \\
\hline Wealth index & & & & & & & \\
\hline Poorest & 1503 & 49.4 & $45 \cdot 5,53 \cdot 2$ & $17 \cdot 0$ & $14 \cdot 8,19 \cdot 5$ & 0.7 & $0.3,1 \cdot 2$ \\
\hline Poor & 1300 & $42 \cdot 3$ & $39 \cdot 2,45 \cdot 5$ & $16 \cdot 5$ & $14 \cdot 3,19 \cdot 1$ & $1 \cdot 2$ & $0.7,2 \cdot 3$ \\
\hline Middle & 1377 & $36 \cdot 2$ & $33 \cdot 3,39 \cdot 2$ & $12 \cdot 7$ & $10 \cdot 2,15 \cdot 7$ & $1 \cdot 2$ & $0 \cdot 7,2 \cdot 3$ \\
\hline Rich & 1411 & $31 \cdot 1$ & $27 \cdot 6,34 \cdot 8$ & $13 \cdot 3$ & $11 \cdot 0,15 \cdot 9$ & $1 \cdot 1$ & $0.6,2.0$ \\
\hline Richest & 1342 & 19.5 & $16 \cdot 8,22 \cdot 5$ & 11.8 & $09.6,14.4$ & 3.6 & $2 \cdot 6,5 \cdot 1$ \\
\hline$P$ value & & & & & & & \\
\hline All & 6933 & $36 \cdot 2$ & $34.4,38.0$ & $14 \cdot 3$ & $13 \cdot 2,15 \cdot 5$ & 1.5 & $1.2,1.9$ \\
\hline
\end{tabular}

*Stunting defined as low height-for-age.

tWasting defined as low weight-for-age.

$\ddagger$ Overweight defined as high weight-for-age.

from the lowest socio-economic condition were more likely to be malnourished (stunting: $49.4 v$. 19.5\%; wasting: $17.0 v \cdot 11 \cdot 8 \%)$ compared with children from the highest socio-economic condition. By age gradient, stunting prevalence increased with child age and the opposite was observed for wasting, which is consistent with many other surveys in different countries ${ }^{(43-45)}$. Undernutrition in children was significantly negatively associated with maternal education. A similar negative association was found for maternal height, place of residence of the household and mother's age at first birth. In contrast, overweight was positively associated with the level of maternal education and socio-economic condition, and its prevalence was higher in urban areas compared with rural areas.

\section{Nutritional status of the motbers}

Table 2 shows that more than one-fifth (22.0\%) of the study respondents (women of reproductive age) were underweight and $18.2 \%$ were overweight. Underweight prevalence was comparatively high among the adolescents (age 15-20 years) and overweight prevalence among the women aged 30-39 years. Rural women were more likely to be underweight (24.2\%) than urban women (15.6\%), whereas urban women were twice as likely to be overweight or obese compared with rural women $(30 \cdot 0 v$ v. $14.3 \%$, respectively). Maternal underweight was negatively associated with level of educational attainment, household socio-economic condition and age at first birth. In contrast, maternal overweight was positively associated with level of education and socio-economic condition, and its prevalence was comparatively low among 
Table 2 Prevalence (\%) of underweight and overweight according to sociodemographic variables among mothers aged 15-49 years (n 6933), Bangladesh, 2014

\begin{tabular}{|c|c|c|c|c|c|c|c|}
\hline \multirow[b]{2}{*}{ Variable } & \multirow[b]{2}{*}{$n$} & \multicolumn{3}{|c|}{ Underweight* } & \multicolumn{3}{|c|}{ Overweight† } \\
\hline & & $\%$ & $95 \% \mathrm{Cl}$ & $P$-value & $\%$ & $95 \% \mathrm{Cl}$ & $P$-value \\
\hline \multicolumn{8}{|l|}{ Place of residence } \\
\hline Urban & 2181 & $15 \cdot 6$ & $12 \cdot 9,18 \cdot 8$ & \multirow[t]{2}{*}{$<0.001$} & $30 \cdot 0$ & $12 \cdot 8,16 \cdot 0$ & \multirow[t]{2}{*}{$<0.001$} \\
\hline Rural & 4752 & $24 \cdot 2$ & $22 \cdot 3,26 \cdot 2$ & & $14 \cdot 3$ & $26 \cdot 9,33 \cdot 2$ & \\
\hline \multicolumn{8}{|l|}{ Mother's age (years) } \\
\hline $15-19$ & 1002 & $32 \cdot 6$ & $29 \cdot 2,36 \cdot 2$ & \multirow[t]{4}{*}{$<0.001$} & $8 \cdot 1$ & $6 \cdot 1,10 \cdot 7$ & \multirow[t]{4}{*}{$<0.001$} \\
\hline $20-29$ & 4271 & $20 \cdot 9$ & $19 \cdot 3,22 \cdot 5$ & & $18 \cdot 0$ & $16 \cdot 4,19 \cdot 8$ & \\
\hline $30-39$ & 1527 & 18.6 & $15 \cdot 6,22 \cdot 1$ & & $25 \cdot 5$ & $21 \cdot 6,29 \cdot 8$ & \\
\hline $40-49$ & 133 & $17 \cdot 9$ & $10 \cdot 5,28 \cdot 6$ & & 18.9 & $12 \cdot 4,27 \cdot 7$ & \\
\hline \multicolumn{8}{|c|}{ Mother's age at first births (years) } \\
\hline$\leq 19$ & 5064 & $24 \cdot 0$ & $22 \cdot 1,26 \cdot 0$ & \multirow[t]{3}{*}{$<0.001$} & $15 \cdot 2$ & $13 \cdot 8,16 \cdot 8$ & \multirow[t]{3}{*}{$<0.001$} \\
\hline $20-29$ & 1806 & $17 \cdot 1$ & $14.8,19 \cdot 6$ & & 25.7 & $22 \cdot 7,29 \cdot 0$ & \\
\hline$\geq 30$ & 63 & $5 \cdot 2$ & $1 \cdot 2,19 \cdot 8$ & & $49 \cdot 3$ & $34.2,64.5$ & \\
\hline \multicolumn{8}{|l|}{ Mother's education } \\
\hline Illiterate & 1071 & $29 \cdot 1$ & $24 \cdot 3,34.5$ & \multirow[t]{4}{*}{$<0.001$} & $10 \cdot 0$ & $7 \cdot 7,12 \cdot 9$ & \multirow[t]{4}{*}{$<0.001$} \\
\hline $1-5$ years of schooling & 1924 & $26 \cdot 8$ & $22 \cdot 9,31 \cdot 0$ & & $12 \cdot 7$ & $10 \cdot 7,15 \cdot 1$ & \\
\hline $6-9$ years of schooling & 3206 & $18 \cdot 9$ & $16 \cdot 8,21 \cdot 2$ & & $21 \cdot 0$ & $19 \cdot 1,22 \cdot 9$ & \\
\hline$\geq 10$ years of schooling & 732 & $10 \cdot 8$ & $8 \cdot 2,14 \cdot 2$ & & 35.5 & $30 \cdot 7,40 \cdot 6$ & \\
\hline \multicolumn{8}{|l|}{ NGO membership } \\
\hline Yes & 2174 & 23.3 & $20 \cdot 3,26 \cdot 5$ & \multirow{2}{*}{$0 \cdot 167$} & 15.4 & $13 \cdot 2,17 \cdot 8$ & \multirow[t]{2}{*}{$<0.001$} \\
\hline No & 4759 & 21.4 & $19 \cdot 7,23 \cdot 3$ & & 19.5 & $17 \cdot 7,21 \cdot 5$ & \\
\hline \multicolumn{8}{|l|}{ Wealth index } \\
\hline Poorest & 1503 & 34.4 & $29 \cdot 6,38 \cdot 7$ & \multirow[t]{6}{*}{$<0.001$} & 5.5 & $4 \cdot 0,7 \cdot 4$ & \multirow[t]{6}{*}{$<0.001$} \\
\hline Poor & 1300 & 28.8 & $25 \cdot 4,32.5$ & & 9.7 & $7 \cdot 8,12 \cdot 0$ & \\
\hline Middle & 1377 & $20 \cdot 0$ & $17.5,22.9$ & & $16 \cdot 4$ & $14.1,19.0$ & \\
\hline Rich & 1411 & $16 \cdot 4$ & $13 \cdot 4,19 \cdot 8$ & & 21.1 & $18 \cdot 2,24.5$ & \\
\hline Richest & 1342 & 8.9 & $7 \cdot 1,11 \cdot 2$ & & $41 \cdot 1$ & $37 \cdot 6,44 \cdot 6$ & \\
\hline All & 6933 & $22 \cdot 0$ & $20 \cdot 4,23 \cdot 7$ & & $18 \cdot 2$ & $16 \cdot 7,19 \cdot 9$ & \\
\hline
\end{tabular}

NGO, non-governmental organization.

*Underweight defined as $\mathrm{BMI}<18.5 \mathrm{~kg} / \mathrm{m}^{2}$.

†Overweight defined as $\mathrm{BMI} \geq 25 \cdot 0 \mathrm{~kg} / \mathrm{m}^{2}$.

women having membership of a non-governmental organization compared with non-members.

\section{Multivariable spatial model}

As discussed earlier, three different models were fitted to each of the outcome measures with the aim of comparing between the models to identify the best one for estimating the district-level prevalence of under- and overnutrition with $95 \%$ credible interval. Model 1 is the standard model with only individual-level predictors; Model 2 consists of the district-level clustering effect in addition to individuallevel predictors; and Model 3 is the extension of Model 2 with additional spatial (neighbouring) effects. We compared the models based on their deviance information criterion to identify the best one with the smallest value. The results (not shown) revealed that Model 3 appeared the best. In addition, the significance of spatial effects (measured using estimated structured variance which is significantly higher than zero) suggested that Model 3 is an appropriate choice for estimating district-level prevalence after adjusting for the individual-level predictors. Therefore, we have summarized the results from Model 3.

\section{Children's under- and overnutrition status}

The results from Model 3 (Table 3) revealed that mother's education, height and age at first birth and household socio-economic condition were significantly associated with each of the nutritional indicators (stunting, wasting and overweight). For example, the negative value of the coefficients (log odds ratio) for mother's education relating to stunting or wasting suggests that increasing level of education decreased the odds of being stunted or wasted, and the positive value relating to overweight suggests that decreasing level of education increased the odds of being overweight. Similarly, mother's height and socio-economic condition were negatively associated with stunting and wasting, and positively associated with childhood overweight. Furthermore, child's age was positively associated with stunting and negatively associated with wasting and overweight. Child's sex showed no significant association with over- and undernutrition.

\section{Women's under- and overnutrition status}

Table 4 shows the associations between women's nutritional status (underweight and overweight) and their individualand household-level covariates. After adjusting for other covariates, household socio-economic condition and mother's education, age and age at first birth were negatively associated with underweight and positively associated with overweight. Women from wealthy households were more likely to be overweight and less likely to be underweight than those from poorer households. Similarly, the likelihood 
Table 3 Adjusted associations between undernutrition (stunting and wasting) and overnutrition (overweight) and sociodemographic variables among children aged 0-59 months, Bangladesh, 2014*

\begin{tabular}{|c|c|c|c|c|c|c|}
\hline \multirow[b]{2}{*}{ Variable } & \multicolumn{2}{|c|}{ Stunting } & \multicolumn{2}{|c|}{ Wasting } & \multicolumn{2}{|c|}{ Overweight } \\
\hline & Coefficient & $95 \% \mathrm{Crl}$ & Coefficient & $95 \% \mathrm{Crl}$ & Coefficient & $95 \% \mathrm{Crl}$ \\
\hline Intercept & 12.573 & $11.016,14.147$ & 1.374 & $-0.512,3.254$ & -8.308 & $-13 \cdot 873,-2 \cdot 784$ \\
\hline Child's age (months) & 0.019 & $0.015,0.022$ & -0.007 & $-0.011,-0.003$ & -0.006 & $-0.018,0.005$ \\
\hline \multicolumn{7}{|l|}{ Child's sex } \\
\hline Male & 0 & - & 0 & - & 0 & - \\
\hline Female & -0.098 & $-0.203,0.008$ & -0.129 & $-0.265,0.007$ & -0.043 & $-0.434,0.344$ \\
\hline Mother's age at first birth (years) & -0.024 & $-0.042,-0.006$ & -0.010 & $-0.034,0.013$ & 0.021 & $-0.041,0.078$ \\
\hline Mother's height $(\mathrm{cm})$ & -0.084 & $-0.094,-0.074$ & -0.017 & $-0.029,-0.004$ & 0.020 & $-0.016,0.056$ \\
\hline \multicolumn{7}{|l|}{ First birth } \\
\hline No & 0 & - & 0 & - & 0 & - \\
\hline Yes & 0.007 & $-0.107,0.121$ & 0.064 & $-0.082,0.209$ & $-0 \cdot 104$ & $-0.517,0.301$ \\
\hline \multicolumn{7}{|l|}{ Mother's education } \\
\hline Illiterate & 0 & - & 0 & - & 0 & - \\
\hline $1-5$ years of schooling & -0.042 & $-0.204,0.119$ & 0.098 & $-0.112,0.312$ & 0.294 & $-0.483,1.176$ \\
\hline $6-9$ years of schooling & -0.268 & $-0.432,-0.104$ & -0.013 & $-0.229,0.205$ & 0.362 & $-0.379,1.227$ \\
\hline$\geq 10$ years of schooling & -0.389 & $-0.653,-0.128$ & 0.043 & $-0.288,0.369$ & 0.764 & $-0.116,1.739$ \\
\hline \multicolumn{7}{|l|}{ Place of residence } \\
\hline Rural & 0 & - & 0 & - & 0 & - \\
\hline Urban & 0.140 & $0.007,0.273$ & -0.168 & $-0.344,0.005$ & -0.056 & $-0.523,0.405$ \\
\hline \multicolumn{7}{|l|}{ Wealth index } \\
\hline Poor & 0 & - & 0 & - & 0 & - \\
\hline Poorer & -0.312 & $-0.473,-0.152$ & -0.036 & $-0.238,0.164$ & 0.135 & $-0.660,0.937$ \\
\hline Middle & -0.394 & $-0.560,-0.228$ & -0.224 & $-0.439,-0.011$ & 0.386 & $-0.352,1.165$ \\
\hline Richer & -0.698 & $-0.877,-0.52$ & -0.374 & $-0.606,-0.143$ & -0.042 & $-0.876,0.799$ \\
\hline Richest & $-1 \cdot 251$ & $-1.473,-1.031$ & -0.434 & $-0.712,-0.159$ & 1.213 & $0.462,2.023$ \\
\hline Unstructured random effect & 0.095 & $0.192,0.048$ & 0.125 & $0.266,0.056$ & 0.009 & $0.046,0.004$ \\
\hline Structured random effect & 0.291 & $0.442,0.191$ & 0.150 & $0.358,0.061$ & 0.165 & $0.667,0.063$ \\
\hline DIC & $8179 \cdot 12$ & & $5658 \cdot 81$ & & $1065 \cdot 54$ & \\
\hline
\end{tabular}

Crl, credible interval; DIC, deviance information criterion.

${ }^{*}$ Spatial model adjusting for both the district-level clustering and spatial (neighbouring) effects in addition to individual-level risk factors.

Table 4 Adjusted associations between undernutrition $\left(\mathrm{BMl}<18.5 \mathrm{~kg} / \mathrm{m}^{2}\right)$ and overweight $\left(\mathrm{BMI} \geq 25.0 \mathrm{~kg} / \mathrm{m}^{2}\right)$ and sociodemographic variables among mothers aged $15-49$ years, Bangladesh, 2014*

\begin{tabular}{|c|c|c|c|c|}
\hline \multirow[b]{2}{*}{ Variable } & \multicolumn{2}{|c|}{ Underweight } & \multicolumn{2}{|c|}{ Overweight } \\
\hline & Coefficient & $95 \% \mathrm{Crl}$ & Coefficient & $95 \% \mathrm{Crl}$ \\
\hline Intercept & 1.196 & $0.741,1.656$ & $-5 \cdot 166$ & $-5.692,-4.648$ \\
\hline Mother's age (years) & -0.060 & $-0.072,-0.048$ & 0.078 & $0.066,0.091$ \\
\hline Mother's age at first birth (years) & -0.002 & $-0.025,0.021$ & -0.004 & $-0.026,0.018$ \\
\hline \multicolumn{5}{|l|}{ Mother's education } \\
\hline Illiterate & 0 & - & 0 & - \\
\hline $1-5$ years of schooling & -0.353 & $-0.531,-0.174$ & 0.420 & $0.168,0.677$ \\
\hline $6-9$ years of schooling & -0.519 & $-0.706,-0.331$ & 0.679 & $0.431,0.934$ \\
\hline$\geq 10$ years of schooling & -0.702 & $-1.022,-0.390$ & 0.725 & $0.415,1.038$ \\
\hline \multicolumn{5}{|l|}{ Place of residence } \\
\hline Rural & 0 & - & 0 & - \\
\hline Urban & -0.049 & $-0.203,0.105$ & 0.248 & $0.095,0.400$ \\
\hline \multicolumn{5}{|l|}{ NGO membership } \\
\hline No & 0 & - & 0 & - \\
\hline Yes & 0.023 & $-0.109,0.154$ & -0.064 & $-0.215,0.086$ \\
\hline \multicolumn{5}{|l|}{ Wealth index } \\
\hline Poor & 0 & - & 0 & - \\
\hline Poorer & -0.345 & $-0.513,-0.177$ & 0.531 & $0.245,0.822$ \\
\hline Middle & -0.686 & $-0.869,-0.506$ & 0.993 & $0.724,1.270$ \\
\hline Richer & -0.850 & $-1.051,-0.652$ & 1.269 & $1.001,1.546$ \\
\hline Richest & -1.473 & $-1 \cdot 742,-1 \cdot 210$ & $2 \cdot 062$ & $1 \cdot 776,2.356$ \\
\hline Unstructured random effect & 0.114 & $0.233,0.056$ & 0.114 & $0.236,0.051$ \\
\hline Structured random effect & 0.365 & $0.561,0.232$ & 0.232 & $0.438,0.121$ \\
\hline DIC & 6843.84 & & $5848 \cdot 78$ & \\
\hline
\end{tabular}

Crl, credible interval; NGO, non-governmental organization; DIC, deviance information criterion.

${ }^{*}$ Spatial model adjusting for both the district-level clustering and spatial (neighbouring) effects in addition to individual-level risk factors.

of being underweight was higher among young women than older ones; and the opposite scenario was observed for overweight. Although membership of a non-governmental organization was found to be significantly associated with over- and undernutrition, it was insignificant when adjusting for the other factors in the multivariable model. 

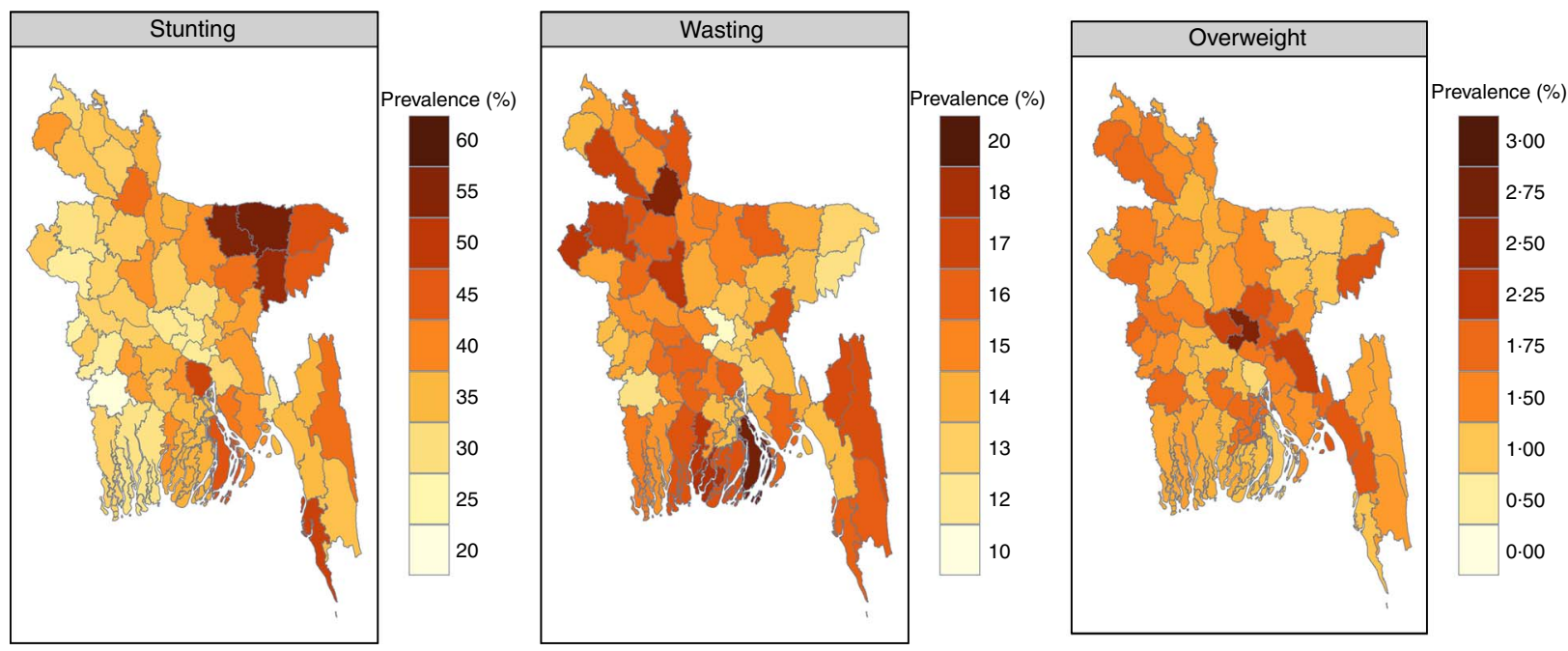

Fig. 1 (colour online) Posterior prevalence of stunting, wasting and overweight among children aged 0-59 months from a Bayesian spatial model, Bangladesh, 2014
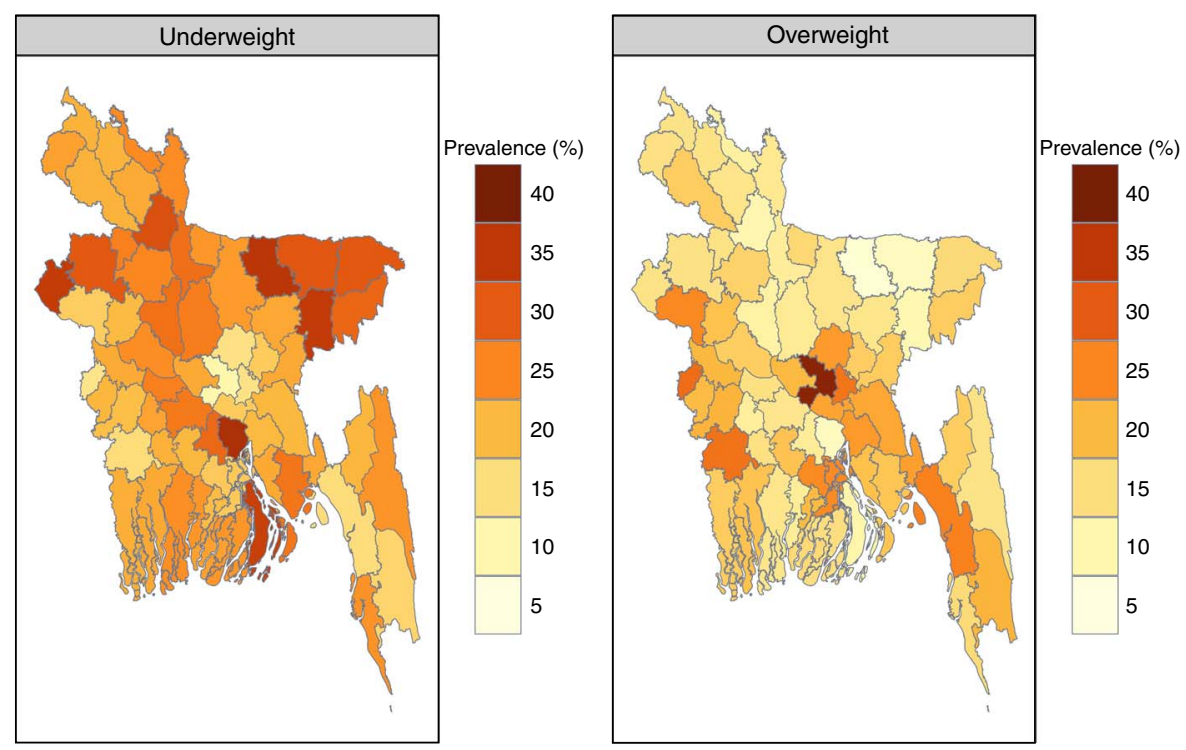

Fig. 2 (colour online) Posterior prevalence of underweight and overweight among mothers aged 15-49 years from a Bayesian spatial model, Bangladesh, 2014

\section{Mapping the prevalence of children's under- and overnutrition}

Figure 1 presents the district-level estimated posterior prevalence (obtained from individual-level predicted posterior probability from the model) of stunting, wasting and overweight among children aged $<5$ years. Stunting prevalence in Bangladesh's districts ranged from $20.3 \%$ in Jessore to $56 \cdot 2 \%$ in Sunamganj and wasting prevalence ranged from $10 \cdot 6 \%$ in Dhaka to $19 \cdot 2 \%$ in Bhola. For child overweight, Shariatpur district $(0 \cdot 8 \%)$ had the least proportion and Dhaka district (2.6\%) had the highest proportion. The map shows that stunting and wasting were more prevalent in the districts of the north-east border of Bangladesh under Sylhet division, and in the Cox's Bazar district (coastal zone) and hilly districts of
Chittagong division. There was very low variability observed in the prevalence of children with overweight between the districts; however, industrially more advanced districts had somewhat more overweight children.

\section{Mapping the prevalence of women's under-and overnutrition}

Figure 2 shows the posterior prevalence of women with underweight and overweight obtained from a Bayesian spatial model. The underweight prevalence ranged from $10 \cdot 1 \%$ in Dhaka to $36 \cdot 2 \%$ in Shariatpur. The districts of Sylhet division were observed to have the highest underweight prevalence while Dhaka and Rajshahi districts were found to have the lowest prevalence. Maternal overweight was least prevalent in Netrokona district $(6 \cdot 2 \%)$, whereas 
the prevalence was highest in Dhaka district (38.6\%). Women in some districts of Dhaka and Chittagong divisions were more likely to overweight, whereas women in most of the districts from Rangpur division were less likely to be overweight.

\section{Discussion}

The current study investigated the association of individuallevel factors with both maternal and child over- and undernutrition in Bangladesh, and further examined regional variation with the aim to identify districts (regions) with high prevalence so that evidence-based policy can be made for quick improvement. Results showed that maternal and childhood under- and overnutrition are significantly associated with sociodemographic factors and geographical location. These findings are similar to those of other studies conducted in recent years in Bangladesh and other lowmiddle-income countries ${ }^{(25,46-51)}$. The striking finding of the current study is the large variation in undernutrition prevalence across the districts of the country. This result could help to recognize the regions where improvement is urgently required.

Findings extracted from the present study could have important implications. As explained in several studies, maternal education plays a significant role in child health outcomes $^{(46,52)}$. This is because educated mothers have greater knowledge on health and nutrition of their children and the ability to recognize illness and seek treatment. Conversely, women with low education are mainly from more impoverished families in general, consume low-quality foods, suffer unhygienic living conditions and have limited access to health services, which in turn affects the health and nutritional status of both these mothers and their children. Moreover, women from poorer families are more likely to get married and give birth at a younger age (often less than 20 years), which in turn affects their nutritional status and that of their children too. In addition, even if they are educated, they often cannot afford the cost of providing nutritious foods and receiving quality health care. In contrast, the likelihood of being overweight is higher among children from the wealthiest households and whose mothers are highly educated. The probable reason is that mothers with high education and better socio-economic conditions are more aware of feeding their children extra nutritious foods without minimum physical activities to compensate. In addition to that, these families are living in the metropolitan area where there is very limited playing space available for children, which in turn affects children's health by increasing their body weight.

Bangladesh has made remarkable progress in improving women's education and socio-economic conditions in general in the last two decades, yet the progress towards reducing both maternal and child undernutrition has been disappointingly plodding ${ }^{(53)}$. In Bangladesh, stunting prevalence has been decreased by 17 percentage points between 2004 and 2014, while there has been minimal variation in wasting ${ }^{(14)}$. This is probably due to the regional differences in undernutrition prevalence, whereby the regions (districts) with very high prevalence may halt the national-level progress. As reported in the current study, there are about seven districts having high prevalence of children with stunting and wasting even compared with the countries reported as having the highest national-level rate among developing countries ${ }^{(54)}$. In addition, there are twelve districts with high stunting prevalence and thirty-two districts with high wasting prevalence, and the prevalence of both indicators is even higher than the respective WHO critical threshold level ${ }^{(35)}$. Similarly, fifty-three districts in total are found to have maternal underweight prevalence higher than the national level. On the other hand, regional variation also exists in the prevalence of women with overnutrition. The estimated prevalence of overweight women in twenty-four districts is found to be greater than the national estimate, while the other districts have lower prevalence than the national prevalence. Similar regional variations have been reported in other studies conducted in India, where there is an increasing concentration of child malnourishment in certain geographical areas ${ }^{(55)}$. Interestingly, malnutritionprone regions still do exist in countries like the USA and Malaysia ${ }^{(56)}$.

The districts with high prevalence of undernutrition identified by mapping can be linked to their poverty levels. The maps show that districts from the four poverty 'hot spots' identified by the Bangladesh Bureau of Statistics experience a high level of maternal and child undernutrition. For example, districts of the haor basin (Netrokona, Sunamgon, Habigonj, Moulvibazar and Sylhet) and coastal belt (Cox's Bazar, Patuakhali and Bhola), a poverty 'hot spot', were found to have very high maternal and childhood undernutrition. Similarly, monga-prone northwestern districts (Faridpir, Gaibandha, Kurigram, Shariatpur, Lalmonirhat and Nilphamari), where there is severe food insecurity during the pre-harvest time, and hilly districts of south-east Bangladesh are also recognized as poverty 'hot spots' of the country having high undernutrition prevalence. The findings have several implications. Women of the haor areas are particularly disadvantaged and vulnerable. Mothers and children living in these regions remain isolated with poor communications and transportation, leading to limited access to health facilities and other services such as education and employment ${ }^{(57)}$. Research shows that coverage of the Expanded Program on Immunization (which provides immunization, vitamin $\mathrm{A}$ and zinc supplements) and exclusive breast-feeding practices are low in these regions, which subsequently affects the nutritional status of women and children ${ }^{(58,59)}$. Moreover, iodine deficiency is higher among people from the coastal belt due to their consumption of locally produced non-iodized salt 
that could have a negative impact on nutrition ${ }^{(60)}$. These districts where undernourishment is most prevalent are also considered one of the food-insecure and vulnerable regions in the context of climate change and natural disasters. Further, low female literacy and primary-school enrolment rates in these areas are likewise associated with the high level of undernutrition. In contrast, overweight is less prevalent in these vulnerable districts; instead, it is concentrated in the districts with better socio-economic conditions, especially in the industrialized districts. Overweight among women of reproductive age has shown an eightfold increase in the past two decades, and this increase has mostly happened in urban areas where industrialization is proceeding rapidly ( $9 \%$ in 1996 to $27.2 \%$ in 2014) ${ }^{(14)}$. The increase may be a result of food habits and lifestyles with increased access to energy-dense foods ${ }^{(54)}$.

The present study's findings raise the need for effective interventions and programmes that could contribute to the greater improvement in undernutrition in lagging districts. Even more potent would be significant policy reform to revitalize the entire health system for local-level tackling of undernutrition. Although the programme is based on a robust design tailored to local needs, it covers only about $30 \%$ of the total population and its efficacy has been questioned $^{(61-63)}$. Besides, management of nutrition in the aftermath of natural disasters and stabilized prices for foods should also be prioritized. The findings of the study suggest the need for implementing district-level planning for nutrition, which is yet to happen, with a special emphasis on high-prevalence districts. Many development partners are implementing nutritional intervention programmes; however, interventions are yet to be implemented at various scales for reaching the entire population. The multisectoral responsibilities should also be accountable for managing district-level inequalities, targeting vulnerable women and people in the ecologically unfavourable parts of the country to improve the coverage of basic and effective nutrition interventions, including exclusive breast-feeding, appropriate complementary feeding and supplementation of micronutrients to children and pregnant/lactating women. Local programmes need promotion of social and economic empowerment and education of women - elements that could have been key to the success of similar and even poorer countries in the region ${ }^{(64,65)}$. Social safety net programmes, such as the Vulnerable Group Feeding programme, should also include cash or food support to address both overt and hidden hunger.

\section{Limitations of the study}

To the best of our knowledge, the current study is the first district-level analysis of nutrition and its key determinants in a low-income country based on a nationally representative survey. Despite this, some limitations must be recognized. We did not have data about gestational age, birth weight of the child and household food security in the study, which would have allowed a more systematic analysis of mother-child linkages ${ }^{(1,66)}$. Household food security is considered an important predictor of undernutrition in both mothers and children. Although the BDHS collected such data previously, it is expected that this survey may not insufficient to ensure whether the household is in a secure position regarding the availability of food or not. Therefore, we recommend a further study should be designed to assess the food security of households, aligned with the set of questions developed by the US Department of Agriculture ${ }^{(67)}$. The present study could not address trends over time at the district level because the previous BDHS did not include rich geographical information. Another potential limitation is use of an indirect measure of household wealth, because in developing countries like Bangladesh it is hard to obtain reliable income and expenditure data and identify household food and non-food expenses. Moreover, the study could not consider the existence or coverage of relevant nutrition programmes at the district level as an independent variable due to the lack or unavailability of reliable data. Despite these limitations, examining the key risk factors of stunted children and overweight mothers is a critical step in developing nutrition intervention programmes that target both extremes of the over- and undernutrition paradox.

\section{Conclusion}

Childhood and maternal undernutrition concentrated in the central, northern and eastern regions in Bangladesh, the geographically unfavourable areas. Under- and overnutrition showed opposite directions, and the districts with high prevalence of overnutrition had low prevalence of undernutrition and vice versa. Therefore, effective intervention programmes need to be designed urgently, focusing on children and women at the grass-roots level of such regions, for controlling the extremes of the overand undernutrition paradox. Simultaneously, integrated programmes for improving education and socio-economic conditions of the general population in the highly concentrated undernutrition regions are also highly required. Failure to do so will ultimately harm the commitment set for all forms of malnutrition by 2030, as part of Sustainable Development Goal 2, to 'end hunger, achieve food security and improved nutrition and promote sustainable agriculture'.

\section{Acknowledgements}

Acknowledgements: The authors acknowledge the National Institute of Population Research and Training (NIPORT), Mitra and Associates and IFC International for proving the open-access data sets used in the present study. icddr,b is grateful to the Government of the People's 
Republic of Bangladesh; the Department of Foreign Affairs, Trade and Development (DFATD), Canada; the Swedish International Development Cooperation Agency (Sida); and the Department for International Development (UK Aid), UK for providing core/unrestricted support. Financial support: This research received no specific grant from any funding agency in the public, commercial or notfor-profit sectors. The authors conducted this study based on their own interest and used data from a secondary source. Conflict of interest: The authors declare that they have no competing interests. Authorship: M.N.M. and M.S.R. conceived of and designed the study. M.N.M., M.S.R. and P.K.R. performed the experiments. M.N.M., M.S.R. and P.K.R. analysed the data. M.N.M. and M.S.R. wrote the paper. M.N.M., M.S.R. and P.K.R. provided critical review, input and revision of the manuscript. All authors read and approved the final manuscript. Ethics of buman subject participation: Not applicable.

\section{References}

1. Black RE, Victora CG, Walker SP et al. (2013) Maternal and child undernutrition and overweight in low-income and middle-income countries. Lancet 382, 427-451.

2. Grantham-McGregor S, Cheung YB, Cueto S et al. (2007) Developmental potential in the first 5 years for children in developing countries. Lancet 369, 60-70.

3. Victora CG, Adair L, Fall C et al. (2008) Maternal and child undernutrition: consequences for adult health and human capital. Lancet 371, 340-357.

4. Pelletier DL, Frongillo EA Jr \& Habicht J-P (1993) Epidemiologic evidence for a potentiating effect of malnutrition on child mortality. Am J Public Health 83, 1130-1133.

5. Pelletier DL \& Frongillo EA (2003) Changes in child survival are strongly associated with changes in malnutrition in developing countries. J Nutr 133, 107-119.

6. United Nations System Standing Committee on Nutrition (2004) 5th report on the world nutrition situation: Nutrition for improved development outcomes. https://www.unscn. org/web/archives_resources/files/rwns5.pdf (accessed July 2017).

7. Bhutta ZA, Das JK, Rizvi A et al. (2013) Evidence-based interventions for improvement of maternal and child nutrition: what can be done and at what cost? Lancet 382, 452-477.

8. Bredenkamp C, Buisman LR \& Van de Poel E (2014) Persistent inequalities in child undernutrition: evidence from 80 countries, from 1990 to today. Int J Epidemiol 43, $1328-1335$.

9. de Onis M, Frongillo EA \& Blössner M (2000) Is malnutrition declining? An analysis of changes in levels of child malnutrition since 1980. Bull World Health Organ 78, 1222-1233.

10. Tzioumis E, Kay MC, Bentley ME et al. (2016) Prevalence and trends in the childhood dual burden of malnutrition in low- and middle-income countries, 1990-2012. Public Health Nutr 19, 1375-1388.

11. Ebbeling CB, Pawlak DB \& Ludwig DS (2002) Childhood obesity: public-health crisis, common sense cure. Lancet 360, 473-482.

12. World Bank (2017) Global Database on Child Growth and Malnutrition. http://www.data.worldbank.org/indicator/SH. STA.STNT.ZS?locations=BD (accessed May 2017).
13. Victora CG, Requejo JH, Barros AJ et al. (2016) Countdown to 2015: a decade of tracking progress for maternal, newborn, and child survival. Lancet 387, 2049-2059.

14. National Institute of Population Research and Training, Mitra and Associates, \& IFC International (2016) Bangladesh Demographic and Health Survey 2014. Dhaka: NIPORT, Mitra and Associates, and IFC International.

15. Sack DA (2008) Achieving the Millennium Development Goals for health and nutrition in Bangladesh: key issues and interventions - an introduction. J Health Popul Nutr 26, 253-260.

16. International Institute for Population Sciences (1998-1999) National Family Health Survey (NFHS-2). https://www. dhsprogram.com/pubs/pdf/FRIND2/FRIND2.pdf (accessed July 2017).

17. International Institute for Population Sciences (2015-2016) National Family Health Survey (NFHS-4). https://dhsprogram. com/pubs/pdf/FR338/FR338.BR.pdf (accessed July 2017).

18. Ministry of Health Nepal, New ERA \& ICF (2017) Nepal 2016 Demographic and Health Survey: Key Findings. Kathmandu: Ministry of Health Nepal.

19. Meshram I, Balakrishna N, Sreeramakrishna K et al. (2016) Trends in nutritional status and nutrient intakes and correlates of overweight/obesity among rural adult women $(\geq 18-60$ years) in India: National Nutrition Monitoring Bureau (NNMB) national surveys. Public Health Nutr 19, $767-776$.

20. Bleich SN, Vercammen KA, Zatz LY et al. (2018) Interventions to prevent global childhood overweight and obesity: a systematic review. Lancet Diabet Endocrinol 6, 332-346.

21. Islam MA, Rahman MM \& Mahalanabis D (1994) Maternal and socioeconomic factors and the risk of severe malnutrition in a child: a case-control study. Eur J Clin Nutr 48, 416-424.

22. Dewey KG \& Cohen RJ (2007) Does birth spacing affect maternal or child nutritional status? A systematic literature review. Matern Child Nutr 3, 151-173.

23. Campbell CC (1991) Food insecurity: a nutritional outcome or a predictor variable? J Nutr 121, 408-415.

24. Ramakrishnan U (2004) Nutrition and low birth weight: from research to practice. Am J Clin Nutr 79, 17-21.

25. Rahman MS, Howlader T, Masud MS et al. (2016) Association of low-birth weight with malnutrition in children under five years in Bangladesh: do mother's education, socioeconomic status, and birth interval matter? PLoS One 11, $\mathrm{e} 0157814$.

26. Mohsena M, Goto R \& Nicholas Mascie-Taylor CG (2015) Regional variation in maternal and childhood undernutrition in Bangladesh: evidence from demographic and health surveys. WHO South East Asia J Public Health 4, 139-149.

27. Bangladesh Bureau of Statistics (2016) Statistical Pocket Book of Bangladesh 2015, p. 8. Dhaka: BBS.

28. Wyatt SB, Winters KP \& Dubbert PM (2006) Overweight and obesity: prevalence, consequences, and causes of a growing public health problem. Am J Med Sci 331, 166-174.

29. Deurenberg P, Yap M \& Van Staveren WA (1998) Body mass index and percent body fat: a meta analysis among different ethnic groups. Int J Obes Relat Metab Disord 22, 1164-1171.

30. United Nations (2017) World Population Prospects: The 2017 Revision. New York: UN.

31. Finance Division, Ministry of Finance (2016) Bangladesh Economic Review 2015. Dhaka: Finance Division, Ministry of Finance.

32. Bangladesh Bureau of Statistics (2010) Report on Monitoring of Employment Survey 2009. Dhaka: BBS.

33. Bangladesh Bureau of Statistics (2012) Report of the Household Income and Expenditure Survey 2010. Dhaka: BBS.

34. Spray AL, Eddy B, Hipp JA et al. (2013) Spatial analysis of undernutrition of children in Leogane Commune, Haiti. Food Nutr Bull 34, 444-461. 
35. World Health Organization (2006) WHO Child Growth Standards: Length/Height-for-Age, Weight-for-Age, Weightfor-Length, Weight-for-Height and Body Mass Index-forAge: Methods and Development. Geneva: WHO.

36. World Health Organization (1995) Physical Status: The Use and Interpretation of Antbropometry. Report of a WHO Expert Committee. WHO Technical Report Series no. 854. Geneva: WHO.

37. Filmer D \& Pritchett LH (2001) Estimating wealth effects without expenditure data - or tears: an application to educational enrollments in states of India. Demography $\mathbf{3 8}$ 115-132.

38. Rutstein SO \& Johnson K (2004) DHS Comparative Report 6: The DHS Wealth Index. Calverton, MD: ORC Macro.

39. Vyas S \& Kumaranayake L (2006) Constructing socioeconomic status indices: how to use principal components analysis. Health Policy Plan 21, 459-468.

40. Howe LD, Hargreaves JR \& Huttly SR (2008) Issues in the construction of wealth indices for the measurement of socio-economic position in low-income countries. Emerg Themes Epidemiol 5, 3.

41. Besag J (1974) Spatial interaction and the statistical analysis of lattice systems. J R Stat Soc Series B Methodol 36, 192-236.

42. Banerjee S, Carlin BP \& Gelfand AE (2014) Hierarchical Modeling and Analysis for Spatial Data. Boca Raton, FL: CRC Press/Chapman \& Hall

43. Kandala N-B, Madungu TP, Emina JB et al. (2011) Malnutrition among children under the age of five in the Democratic Republic of Congo (DRC): does geographic location matter? BMC Public Health 11, 261.

44. Semba RD, de Pee S, Sun K et al. (2008) Effect of parental formal education on risk of child stunting in Indonesia and Bangladesh: a cross-sectional study. Lancet $\mathbf{3 7 1}$, 322-328.

45. Mengistu K, Alemu K \& Destaw B (2013) Prevalence of malnutrition and associated factors among children aged 6-59 months at Hidabu Abote District, North Shewa, Oromia Regional State. J Nutr Dis Ther T1, 001.

46. Wang CC \& Pies CA (2004) Family, maternal, and child health through photovoice. Matern Child Health J 8, 95-102.

47. Di Cesare M, Bhatti Z, Soofi SB et al. (2015) Geographical and socioeconomic inequalities in women and children's nutritional status in Pakistan in 2011: an analysis of data from a nationally representative survey. Lancet Glob Health 3, e229-e239.

48. Jesmin A, Yamamoto SS, Malik AA et al. (2011) Prevalence and determinants of chronic malnutrition among preschool children: a cross-sectional study in Dhaka City, Bangladesh. J Health Popul Nutr 29, 494-499.

49. Mishra VK \& Retherford RD (2000) Women's education can improve child nutrition in India. Natl Fam Health Surv Bull issue $15,1-4$.

50. Kanjilal B, Mazumdar PG, Mukherjee M et al. (2010) Nutritional status of children in India: household socioeconomic condition as the contextual determinant. Int $J$ Equity Health 9, 19.

51. Bhutta ZA, Gazdar H \& Haddad L (2013) Seeing the unseen: breaking the logjam of undernutrition in Pakistan. IDS Bull 44, 1-9.
52. Desai S \& Alva S (1998) Maternal education and child health: is there a strong causal relationship? Demography 35, 71-81.

53. Das SK, Chisti MJ, Malek MA et al. (2015) Changing childhood malnutrition in Bangladesh: trends over the last two decades in urban-rural differentials (1993-2012). Public Health Nutr 18, 1718-1727.

54. Stevens GA, Finucane MM, Paciorek CJ et al. (2012) Trends in mild, moderate, and severe stunting and underweight, and progress towards MDG 1 in 141 developing countries: a systematic analysis of population representative data. Lancet 380, 824-834.

55. Nair K (2007) Malnourishment among children in India: a regional analysis. Econ Polit Wkly 15 Sept issue, 3797-3803.

56. Slifkin R, Goldsmith L \& Ricketts T (2000) Race and Place: Urban-Rural Difference in Health for Racial and Ethnic Minorities. Working Paper no. 66. Chapel Hill, NC: NC Rural Health Research and Policy Analysis Program.

57. Kandala NB, Fahrmeir L, Klasen S et al. (2009) Geo-additive models of childhood undernutrition in three sub-Saharan African countries. Popul Space Place 15, 461-473.

58. Uddin MJ, Larson CP, Oliveras E et al. (2009) Child immunization coverage in rural hard-to-reach Haor areas of Bangladesh: possible alternative strategies. Asia Pac J Public Health 21, 8-18.

59. Rahman M \& Obaida-Nasrin S (2010) Factors affecting acceptance of complete immunization coverage of children under five years in rural Bangladesh. Salud Publica Mex 52, 134-140.

60. Rasheed S, Siddique A, Sharmin T et al. (2016) Salt intake and health risk in climate change vulnerable coastal Bangladesh: what role do beliefs and practices play? PLOS One 11, e0152783.

61. World Bank (2005) Maintaining Momentum to 2015? An Impact Evaluation of Interventions to Improve Maternal and Child Health and Nutrition in Bangladesh. Washington, DC: World Bank.

62. Nahar S, Mascie-Taylor CG \& Begum HA (2009) Impact of targeted food supplementation on pregnancy weight gain and birth weight in rural Bangladesh: an assessment of the Bangladesh Integrated Nutrition Program (BINP). Public Health Nutr 12, 1205-1212.

63. Hossain SM, Duffield A \& Taylor A (2005) An evaluation of the impact of a US $\$ 60$ million nutrition programme in Bangladesh. Health Policy Plan 20, 35-40.

64. Chowdhury AMR, Bhuiya A, Chowdhury ME et al. (2013) The Bangladesh paradox: exceptional health achievement despite economic poverty. Lancet 382, 1734-1745.

65. El Arifeen S, Christou A, Reichenbach L et al. (2013) Community-based approaches and partnerships: innovations in health-service delivery in Bangladesh. Lancet 382, 2012-2026.

66. Katz J, Lee AC, Kozuki $\mathrm{N}$ et al. (2013) Mortality risk in preterm and small-for-gestational-age infants in low-income and middle-income countries: a pooled country analysis. Lancet 382, 417-425.

67. Bickel G, Nord M, Price C et al. (2000) Guide to Measuring Household Food Security (Revised 2000). Alexandria, VA: US Department of Agriculture, Food and Nutrition Service, Office of Analysis, Nutrition, and Evaluation. 